\title{
Predictive impact of polymorphism of PNPLA3 on HCC development after interferon therapy in Japanese patients with chronic hepatitis $C$
}

\author{
Yuki Moritou ${ }^{1}$, Fusao Ikeda ${ }^{1,2^{*}}$, Yoshiaki Iwasaki ${ }^{3}$, Nobuyuki Baba ${ }^{4}$, Kouichi Takaguchi ${ }^{4}$, Tomonori Senoh ${ }^{4}$, \\ Takuya Nagano ${ }^{4}$, Yasuto Takeuchi ${ }^{1}$, Tetsuya Yasunaka' ${ }^{1}$ Hideki Ohnishi ${ }^{1,2}$, Yasuhiro Miyake ${ }^{1}$, Akinobu Takaki ${ }^{1}$, \\ Kazuhiro Nouso ${ }^{1,2}$ and Kazuhide Yamamoto ${ }^{1,2}$
}

\begin{abstract}
The impact of single-nucleotide polymorphisms (SNP) of patatin-like phospholipase domain-containing protein 3 (PNPLA3) on development of hepatocellular carcinoma (HCC) is not clarified for Japanese patients with chronic hepatitis C. The present study investigated the associations of rs738409 PNPLA3 with HCC development after the antiviral therapy with peg-interferon and ribavirin for Japanese patients with hepatitis $C$ virus serotype 1 and high viral load. Of the 271 patients enrolled in the study, 20 patients developed HCC, during a median follow-up period of 4.6 years. Multivariate analysis in the proportional hazards models revealed that sex, body mass index, platelet counts, and alpha feroprotein (AFP) had significant associations with HCC development ( $p=0.011,0.029,0.0002$, and 0.046, respectively). Multivariate regression analysis revealed that PNPLA3 $148 \mathrm{M}$ was significantly associated with serum AFP level $(p=0.032)$, other than body mass index, platelet count, and alanine aminotransferase $(p=0.0006,0.0002$, and 0.037 , respectively), and that serum AFP level was significantly associated with PNPLA3 $148 \mathrm{M}(p=0.017)$. Serum AFP level is an important factor in predicting HCC development after the antiviral therapy for Japanese patients with chronic hepatitis $C$, the mechanism of which might involve its significant associations with the SNP genotype of PNPLA3.
\end{abstract}

Keywords: PNPLA3, Interferon, HCC, HCV

\section{Introduction}

Hepatitis C virus (HCV) infection causes chronic hepatitis, and may progress to liver cirrhosis and hepatocellular carcinoma (HCC). More than 170 million people worldwide are infected with $\mathrm{HCV}$, creating a serious global health problem (Kato 2001). Combination therapy with pegylated interferon alpha and ribavirin has a sustained virological response (SVR) in $50 \%$ of patients with $\mathrm{HCV}$ genotype 1 (Firpi \& Nelson 2007). Recent therapeutic regimens using direct-acting antiviral agents have improved the SVR up to $80 \%$ (Ghany et al. 2011a; Zeuzem et al. 2011; Kumada et al. 2012).

\footnotetext{
* Correspondence: fikeda@md.okayama-u.ac.jp

'Department of Gastroenterology and Hepatology, Okayama University Graduate School of Medicine, Dentistry and Pharmaceutical Sciences, 2-5-1, Shikata-cho, Okayama 700-8558, Japan

${ }^{2}$ Department of Molecular Hepatology, Okayama University Graduate School of Medicine, Dentistry and Pharmaceutical Sciences, Okayama, Japan Full list of author information is available at the end of the article
}

Recently, the single-nucleotide polymorphism (SNP) of rs738409 patatin-like phospholipase domain-containing protein 3 (PNPLA3) has been identified for its significant associations with liver steatosis and fibrosis in patients with fatty liver disease and alcoholic liver disease (Romeo et al. 2008; Valenti et al. 2010). As for patients with chronic hepatitis C, associations of this SNP genotype with liver steatosis, fibrosis, and the outcome of interferon therapy have also been suggested (Trépo et al. 2011; Valenti et al. 2011). These results were mostly obtained from the analyses of patients of European origins; however, analysis of Japanese patients could yield somehow different results. A recent genome-wide association study for Japanese patients with non-alcoholic fatty liver disease by Kawaguchi et al. revealed that the risk variant of PNPLA3 $148 \mathrm{M}$ is significantly associated with nonalcoholic steatohepatitis, but did not show significant differences in liver steatosis or fibrosis among the patients 
with non-alcoholic fatty liver disease (Kawaguchi et al. 2012). Miyashita et al. studied the associations of the SNP genotype of PNPLA3 with liver fibrosis and inflammation for Japanese patients with chronic hepatitis C, without significant associations in the results (Miyashita et al. 2012). Takeuchi et al. reported that the SNP genotype of PNPLA3 might not affect HCC prognosis in Japanese patients with hepatitis B virus, $\mathrm{HCV}$, or non-alcoholic fatty liver diseases (Takeuchi et al. 2013). Thus, it is possible that ethnic differences exist in the genetic background of the pathogenesis of chronic liver diseases.

Prediction or early detection of HCC development after antiviral therapy is increasingly needed because of recent improvement of antiviral therapy. The present study investigated the associations of the SNP genotype of PNPLA3 with HCC development after interferon therapy in Japanese patients with chronic hepatitis $\mathrm{C}$, in order to clarify the predictive impact of the SNP genotype of PNPLA3 on HCC development in Japanese patients with chronic hepatitis $C$.

\section{Methods \\ Patients}

The present study enrolled 271 Japanese patients suffering from chronic hepatitis $\mathrm{C}$ with HCV serotype 1 and a high viral load $5.0 \log \mathrm{IU} / \mathrm{mL}$ in real-time PCR. All patients received 48 weeks of antiviral therapy with standard doses of pegylated interferon alpha-2a $(180 \mu \mathrm{g} /$ week $)$ or $2 \mathrm{~b}$ (1.5 $\mu \mathrm{g} /$ body weight/week) with ribavirin (600-1000 mg/ day) at either Okayama University Hospital or Kagawa Prefectural Central Hospital. The outcomes of the interferon therapy were evaluated, according to the practice guidelines by the American association for the study of liver diseases, and the Japan society of hepatology (Ghany et al. 2011b). Hepatocellular carcinoma was ruled out using dynamic computed tomography, magnetic resonance imaging or ultrasonography prior to the start of the interferon therapy. Heavy alcohol-drinking behavior was defined as daily alcohol intake $>70$ grams. Patients with hepatitis B virus co-infection, human immunodeficiency virus co-infection, or autoimmune liver disease were not included in the study. The study was performed in accordance with the Helsinki Declaration, and the protocols were approved by the ethics committees of the participating institutes. All patients provided informed consent before enrolment into the study.

\section{Diagnosis of liver histology}

Liver histology was evaluated for 219 patients prior to the start of the interferon therapy. Liver fibrosis stage and hepatitis activity grade were assigned for all patients by two pathologists according to the criteria of Desmet et al. (1994). The severity grade of hepatic steatosis was defined according to the criteria used by Valenti et al. (2011).

\section{Genotyping of single nucleotide polymorphism}

Genomic DNA was extracted from whole-blood samples by means of a QIAamp DNA Mini Kit according to the manufacturer's protocol (Qiagen, Tokyo, Japan). The SNPs of rs8099917 IL28B, and rs738409 PNPLA3 were genotyped using the TaqMan predesigned SNP genotyping assays, as recommended by the manufacturer (Applied Biosystems, Tokyo, Japan). The SNP genotypes of all the samples could be obtained with these systems.

\section{Variations of the amino acid 70 of HCV core}

For the 241 patients in the study, variations of the amino acid 70 of HCV core (arginine or glutamine) were determined with direct sequence method. The amplifications of $\mathrm{HCV}$ core in the serum were performed as previously reported (Ikeda et al. 2010), and direct sequencing was carried out by Big Dye termination cycle sequencing using an ABI Prism 310 genetic analyzer (Applied Biosystems, Foster City, CA).

\section{Follow-up and HCC diagnosis}

In accordance with the clinical practice manual from the Japan Society of Hepatology (Makuuchi et al. 2010), HCC diagnosis was done using ultrasonography, computed tomography, magnetic resonance imaging, hepatic angiography, and/or tumor biopsy, in combination with the detection of serum levels of alpha-fetoprotein (AFP), AFP-L3, and des gamma-carboxy prothrombin (DCP). Only the patients without HCC at 1 year or longer after interferon therapy were included in the study. This follow-up period was defined as the time between the cessation of interferon therapy and HCC diagnosis or the latest confirmation of survival.

\section{Statistical analysis}

Data are expressed as means \pm standard deviations. Proportional hazards models were utilized to identify the factors associated with HCC development during the follow-up period after interferon therapy. The incidences of HCC development were also estimated using the Kaplan-Meier method and compared with the log-rank test. Factors associated with the SNP genotype of PNPLA3 and AFP were analyzed by stepwise logistic regression analysis. A value of $p<0.05$ was considered significant. Statistical analysis was performed with JMP software (SAS Institute, Cary, NC).

\section{Results}

Patient characteristics of the patients enrolled in the study

The characteristics of patients enrolled in the study are shown in Table 1 . Their average age was 57 years, and 131 patients (48\%) were female. Interferon therapy resulted in sustained virological responses in 126 patients (46.5\%), relapse in 77 patients, and null or partial 


\begin{tabular}{|c|c|}
\hline Patient characteristics & $\mathrm{N}=\mathbf{2 7 1}$ \\
\hline Age (years) & $57 \pm 10^{\dagger}$ \\
\hline Sex (male/female) & 140/131 \\
\hline Body mass index & $24 \pm 3^{+}$ \\
\hline Liver fibrosis (Stage 1/2/3/4) & $71 / 82 / 60 / 6$ \\
\hline Hepatitis activity (Grade 1/2/3) & $164 / 49 / 6$ \\
\hline rs 8099917 of IL28B (TT/TG/GG) & 198/72/1 \\
\hline rs 738409 of PNPLA3 (CC/CG/GG) & $81 / 136 / 54$ \\
\hline HCV core amino acids 70 (arginine/glutamine) & $150 / 91$ \\
\hline Alanine aminotransferase (IU/L) & $66 \pm 55^{\dagger}$ \\
\hline Platelet count $\left(10000 / \mathrm{mm}^{3}\right)$ & $17 \pm 6^{+}$ \\
\hline Alpha fetoprotein (ng/mL) & $11 \pm 19^{\dagger}$ \\
\hline HOMA-IR & $4.2 \pm 8.4^{\dagger}$ \\
\hline Therapeutic outcome (SVR/relapse/NVR) & $126 / 77 / 68$ \\
\hline
\end{tabular}

${ }^{\dagger}$ : Mean \pm standard deviation; PNPLA3, patatin-like phospholipase domaincontaining protein 3; HOMA-IR, homeostasis model assessment insulin resistance; SVR, sustained virological response; NVR, null or partial virological response.

virological response (NVR) in 68 patients. During the follow-up after the interferon therapy (median period of 4.6 year), the development of HCC was observed in 20 patients (16\%). The results of AFP-L3 prior to interferon therapy were undetectable or below the lower limit of quantification for all patients. Consistent with previous reports (Makuuchi et al. 2010; He et al. 2009; Nishiguchi et al. 2001), SVR contributed to lowering the risk of HCC development (Hazards ratio 0.13, $p=0.0067$, Table 2), while patients with NVR showed a high incidence of HCC (Hazards ratio 2.4, $p=0.048$ ). The incidences of HCC development were compared among patient groups formed according to interferon therapy outcomes (Figure 1A). The NVR patients had much higher incidence of HCC development than the SVR or relapse patients $(p=0.0055$, the log-rank test). The SVR and relapse patients had similar risks of HCC development during the first 2 years after interferon therapy, while relapse patients had increased risk thereafter.

\section{Patient characteristics associated with HCC development after interferon therapy}

We compared the patient characteristics related to HCC development in proportional hazards models (Table 2). Male gender, high body mass index (BMI), advanced liver fibrosis, low platelet counts, and high levels of AFP had significant associations with HCC development in univariate analysis (Hazards ratio 5.6, $p=0.0062$, Hazards ratio $1.2, p=0.0086$, Hazards ratio $3.8, p<0.0001$, Hazards ratio $0.77, p<0.0001$, and Hazards ratio $1.1, p=0.0002$, respectively). The incidence of HCC development was significantly lower in the patients with PNPLA3 $148 \mathrm{M}$ than in those with PNPLA3 148I (Hazards ratio $0.4, p=0.043$ ). Liver steatosis and amino acid 70 of HCV core affected HCC development slightly (Hazards ratio 2.1, $p=0.067$, and Hazards ratio 3.4, $p=0.082$, respectively), while the SNP genotype of IL28B was not associated with the risk of HCC development (Hazards ratio 0.62, $p=0.31$ ). Stepwise multivariate analysis revealed that male gender, high BMI, low platelet

Table 2 Proportional hazards model for the development of hepatocellular carcinoma

\begin{tabular}{|c|c|c|c|c|}
\hline \multirow[b]{2}{*}{ Factors } & \multicolumn{2}{|l|}{ Univariate analysis } & \multicolumn{2}{|l|}{ Multivariate analysis } \\
\hline & Hazards ratio (range ${ }^{\dagger}$ ) & $p$ & Hazards ratio (range ${ }^{\dagger}$ ) & $p$ \\
\hline Age (years) & $1.0(0.99-1.1)$ & 0.12 & & \\
\hline Sex (1: male) & $5.6(1.6-19)$ & 0.0062 & $5.5(1.5-21)$ & 0.011 \\
\hline Body mass index & $1.2(1.0-1.3)$ & 0.0086 & $1.2(1.0-1.3)$ & 0.029 \\
\hline Liver fibrosis & $3.8(1.9-7.3)$ & $<0.0001$ & & \\
\hline Hepatitis activity & $1.1(0.44-2.6)$ & 0.88 & & \\
\hline Liver steatosis (>30\%) & $2.1(0.95-4.8)$ & 0.067 & & \\
\hline rs 8099917 of IL28B (TT) & $0.62(0.25-1.6)$ & 0.31 & & \\
\hline rs 738409 of PNPLA3 (148 M) & $0.40(0.17-0.97)$ & 0.043 & & \\
\hline Amino acid 70 of HCV core (glutamine) & $3.4(0.86-14)$ & 0.082 & & \\
\hline Alanine aminotransferase (>40 IU/L) & $1.2(0.68-2.2)$ & 0.51 & & \\
\hline Platelet count $\left(10^{4} / \mathrm{mm}^{3}\right)$ & $0.77(0.69-0.87)$ & $<0.0001$ & $0.76(0.66-0.88)$ & 0.0002 \\
\hline Alpha fetoprotein (ng/mL) & $1.1(1.0-1.1)$ & 0.0002 & $1.1(1.0-1.1)$ & 0.046 \\
\hline HOMA-IR & $0.97(0.79-1.2)$ & 0.76 & & \\
\hline Sustained virological response & $0.13(0.03-0.57)$ & 0.0067 & & \\
\hline Null or partial virological response & $2.4(1.0-5.9)$ & 0.048 & & \\
\hline
\end{tabular}

${ }^{\dagger}$ : 95\% confidence interval; PNPLA3, patatin-like phospholipase domain-containing protein 3; HOMA-IR, homeostasis model assessment insulin resistance. 

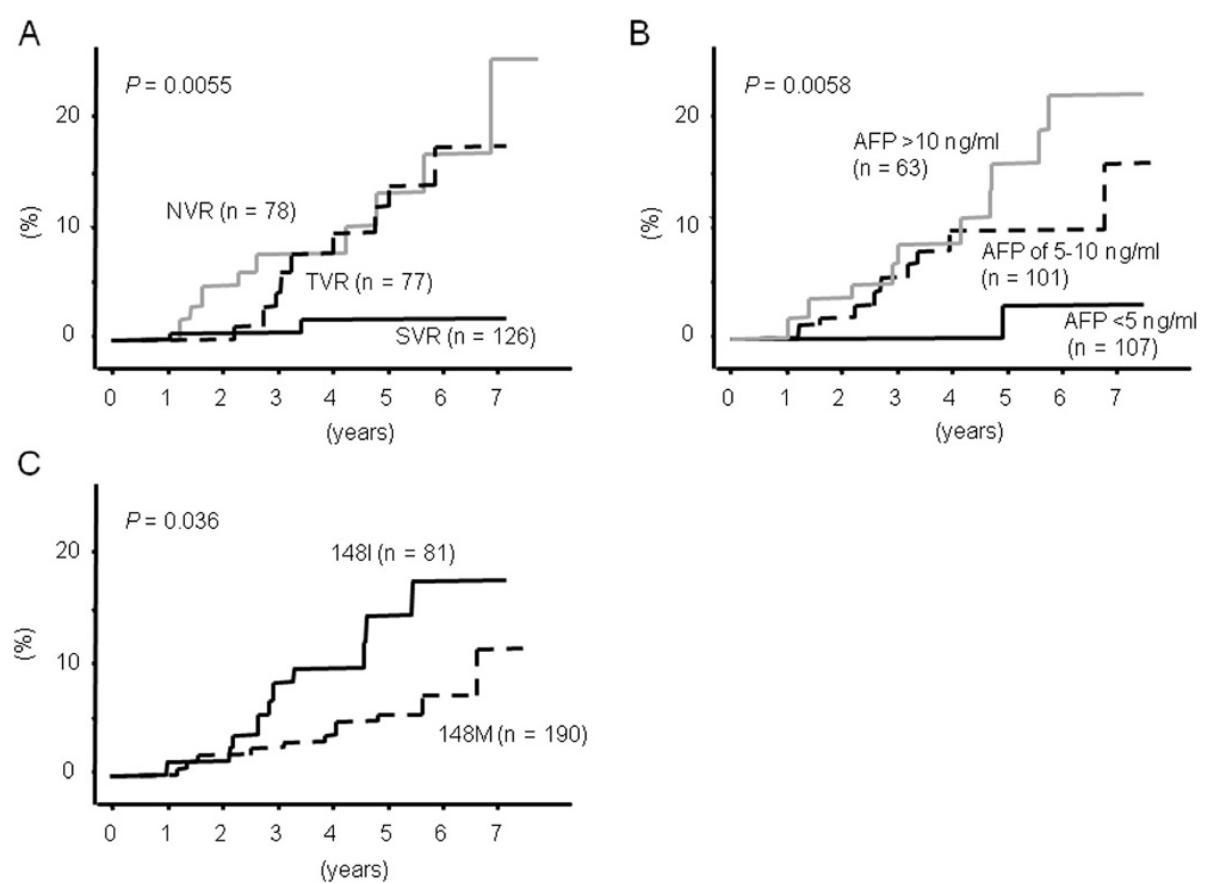

Figure 1 The incidence of HCC development after interferon therapy. The incidence of HCC development after interferon therapy was presumed by the Kaplan-Meier method for each group by dividing the patients by the outcomes of the interferon therapy (A), serum levels of alpha fetoproteins prior to the interferon therapy (B), and the SNP genotypes of PNPLA3 (C).

counts, and high levels of AFP had significant associations with HCC development (Hazards ratio 5.5, $p=0.011$, Hazards ratio $1.2, p=0.029$, Hazards ratio $0.76, p=0.0002$, and Hazards ratio 1.1, $p=0.046$, respectively).

\section{PNPLA3 $148 M$ is independently associated with low levels of AFP}

Serum AFP levels prior to the interferon therapy were significantly associated with HCC development after interferon therapy ( $p=0.0058$, the log-rank test, Figure 1B). Especially, patients with AFP $<5 \mathrm{ng} / \mathrm{mL}$ prior to interferon therapy showed significantly lower incidence of HCC development than those with AFP $\geq 5 \mathrm{ng} / \mathrm{mL}$ (Hazards ratio 0.09, $p=0.021$ ). We further examined the patient characteristics prior to the interferon therapy regarding associations with serum AFP by stepwise logistic regression analysis (Table 3 ). The results showed several significant factors associated with serum AFP, such as BMI, platelet count, and alanine aminotransferase $(p=0.0006$, 0.0002 , and 0.037 , respectively). The SNP genotype of PNPLA3 was also an independent factor associated with serum AFP $(p=0.032)$. Therefore, we studied the patient characteristics associated with the PNPLA3 $148 \mathrm{M}$ genotype (Table 4). Low levels of AFP $>5 \mathrm{ng} / \mathrm{mL}$ were significantly associated with PNPLA3 $148 \mathrm{M}$ in stepwise multivariate logistic regression analysis $(p=0.017)$. Advanced liver fibrosis was also associated with PNPLA3 $148 \mathrm{M}$ in univariate analysis $(p=0.028)$, although this association did not show statistical significance in multivariate analysis $(p=0.20)$.

\section{Discussion}

The impact of the SNP of PNPLA3 on interferon therapy and HCC development after interferon therapy is still undetermined for Japanese patients with chronic hepatitis $C$. The present study investigated the factors associated with HCC development after interferon therapy for Japanese patients with chronic hepatitis $C$, and is the first to clarify significant associations of the SNP of PNPLA3 with HCC development after interferon therapy.

The incidence of HCC development was significantly lower in the patients with PNPLA3 $148 \mathrm{M}$ than in those with PNPLA3 148I (Table 2, and Figure 1C). Considering the roles of the SNP genotype of PNPLA3 with HCVrelated $\mathrm{HCC}$, a previous report by $\mathrm{He}$ et al. revealed impaired triglyceride hydrolysis in those with PNPLA3 $148 \mathrm{M}$ (He et al. 2009), which might work against direct regulation of $\mathrm{HCV}$ in hepatic lipid metabolism, and suppress HCC development. We explored clinical characteristics as they related to the SNP genotype of PNPLA3. In general, advanced liver fibrosis, high age, and male gender have been reported as risk factors of HCC development for patients with chronic hepatitis C (Nishiguchi et al. 2001; Imai et al. 1998; Yoshida et al. 1999). Liver steatosis, inflammation, insulin 
Table 3 Stepwise logistic regression analysis of the factors related to alpha fetoprotein $>5 \mathrm{ng} / \mathrm{mL}$

\begin{tabular}{|c|c|c|c|c|}
\hline \multirow[b]{2}{*}{ Factors } & \multicolumn{2}{|l|}{ Univariate analysis } & \multicolumn{2}{|l|}{ Multivariate analysis } \\
\hline & Odds ratio (range ${ }^{\dagger}$ ) & $p$ & Odds ratio (range ${ }^{\dagger}$ ) & $p$ \\
\hline Age (years) & $1.0(1.0-1.1)$ & 0.0024 & $1.0(1.0-1.1)$ & 0.064 \\
\hline Sex (1:male) & $0.95(0.58-1.6)$ & 0.84 & & \\
\hline Body mass index & $1.2(1.1-1.3)$ & 0.0005 & $1.2(1.1-1.3)$ & 0.0006 \\
\hline Liver fibrosis & $2.8(1.9-4.2)$ & $<0.0001$ & & \\
\hline Hepatitis activity & $1.1(0.61-1.9)$ & 0.83 & & \\
\hline Liver steatosis (1: >30\%) & $1.8(1.1-3.0)$ & 0.026 & $1.3(0.72-2.4)$ & 0.37 \\
\hline rs 8099917 of IL28B (1: TT) & $1.1(0.65-2.0)$ & 0.67 & & \\
\hline rs738409 of PNPLA3 (1: 148 M) & $0.36(0.20-0.65)$ & 0.0007 & $0.46(0.22-0.93)$ & 0.032 \\
\hline Amino acid 70 of HCV core (1: glutamine) & $1.1(0.58-2.2)$ & 0.71 & & \\
\hline Alanine aminotransferase (1: >40 IU/L) & $2.3(1.6-3.3)$ & $<0.0001$ & $1.6(1.0-2.4)$ & 0.037 \\
\hline Platelet count $\left(1:>1.5 \times 10^{5} / \mathrm{mm}^{3}\right)$ & $0.33(0.22-0.50)$ & $<0.0001$ & $0.34(0.19-0.60)$ & 0.0002 \\
\hline HOMA-IR & $1.0(0.97-1.1)$ & 0.33 & & \\
\hline
\end{tabular}

${ }^{\dagger}:$ 95\% confidence interval; PNPLA3, patatin-like phospholipase domain-containing protein 3; HOMA-IR, homeostasis model assessment insulin resistance.

resistance, and excessive body composition may accelerate HCC development. Among those factors, we could find none significantly associated with the SNP genotype of PNPLA3 as previously reported (Miyashita et al. 2012; Kotronen et al. 2009).

Serum AFP was only the factor we found to be significantly associated with the PNPLA3 genotype in the present study; patients with PNPLA3 $148 \mathrm{M}$ had lower levels of serum AFP than those with PNPLA3 148I. Meanwhile, multiple factors such as advanced liver fibrosis, inflammation, and body composition were significantly associated with serum AFP. Only the SNP genotype of PNPLA3 is the significant factor that is genetically determined. The associations of serum AFP with HCC development and the mechanisms by which the SNP genotypes of PNPLA3 contribute to serum AFP are still unknown, and need to be clarified in a future study.
Recent studies on Caucasian patients have shown that PNPLA3 $148 \mathrm{M}$ was significantly associated with liver steatosis (Poynard et al. 2003; Valenti et al. 2012; Clark et al. 2012), while the present study on Japanese patients showed no significant impact of the SNP genotype of PNPLA3 on liver steatosis for the patients with chronic hepatitis C. The high distribution of PNPLA3 $148 \mathrm{M}$ in Japanese patients might be associated with ethnic differences in the role of PNPLA3 in the pathogenesis of chronic liver disease; in the Hap Map study the prevalence of PNPLA3 $148 \mathrm{M}$ was found to be $43 \%$ in the Japanese, much higher than its prevalence in Caucasians (23\%).

In conclusion, serum AFP level is an important factor in predicting $\mathrm{HCC}$ development after the antiviral therapy for Japanese patients with chronic hepatitis $\mathrm{C}$, the mechanism of which might involve its significant associations with the SNP genotype of PNPLA3.

Table 4 Stepwise logistic regression analysis of the factors related to PNPLA3 148 M

\begin{tabular}{|c|c|c|c|c|}
\hline \multirow[b]{2}{*}{ Factors } & \multicolumn{2}{|l|}{ Univariate analysis } & \multicolumn{2}{|l|}{ Multivariate analysis } \\
\hline & Odds ratio (range ${ }^{\dagger}$ ) & $p$ & Odds ratio $\left(\right.$ range $^{\dagger}$ ) & $p$ \\
\hline Age (years) & $0.98(0.95-1.0)$ & 0.12 & & \\
\hline Sex (1: male) & $0.69(0.41-1.2)$ & 0.17 & & \\
\hline Body mass index & $1.0(0.93-1.1)$ & 0.73 & & \\
\hline Liver fibrosis & $0.68(0.48-0.96)$ & 0.028 & $0.78(0.54-1.1)$ & 0.20 \\
\hline Hepatitis activity & $1.3(0.70-2.3)$ & 0.43 & & \\
\hline Liver steatosis (1: >30\%) & $0.96(0.55-1.7)$ & 0.87 & & \\
\hline Amino acid 70 of HCV core (1: glutamine) & $0.80(0.38-1.7)$ & 0.56 & & \\
\hline Alanine aminotransferase $(1:>40 \mathrm{IU} / \mathrm{L})$ & $0.72(0.51-1.0)$ & 0.068 & & \\
\hline Platelet count $\left(1:>1.5 \times 10^{5} / \mathrm{mm}^{3}\right)$ & $2.1(0.93-4.7)$ & 0.076 & & \\
\hline Alpha fetoprotein (1: >5 ng/mL) & $0.36(0.20-0.65)$ & 0.0007 & $0.42(0.21-0.85)$ & 0.017 \\
\hline HOMA-IR & $0.18(0.92-1.0)$ & 0.18 & & \\
\hline
\end{tabular}

\footnotetext{
${ }^{\dagger}:$ 95\% confidence interval; PNPLA3, patatin-like phospholipase domain-containing protein 3; HOMA-IR, homeostasis model assessment insulin resistance.
} 


\section{Abbreviations}

HCV: Hepatitis C virus; HCC: Hepatocellular carcinoma; SVR: Sustained virological response; SNP: Single-nucleotide polymorphisms; PNPLA3: Patatin-like phospholipase domain-containing protein 3; AFP: Alpha fetoprotein; DCP: Des gamma-carboxy prothrombin; NVR: Null or partial virological response; BMl: Body mass index.

\section{Competing interests}

The authors declare that no competing interests exist.

\section{Authors' contributions}

YM and FI designed the project, carried out research and drafted the manuscript. YW contributed to project design, and revised the drafted manuscript. NB, KT, TS, TN, YT, TY, HO, YM, AT, and KN contributed to data collections. KY contributed to project design. All authors read and approved the final manuscript.

\section{Acknowledgements}

This work was supported in part by the Research Program of Intractable Disease sponsored by the Ministry of Health, Labor, and Welfare of Japan, and by a Grant-in-Aid (22590733) for Scientific Research from the Ministry of Education, Culture, Sports, Science and Technology of Japan (to Y. I.). We thank Chizuru Mori for technical assistance and Toshie Ishi for valuable help in data collection.

\section{Author details}

${ }^{1}$ Department of Gastroenterology and Hepatology, Okayama University Graduate School of Medicine, Dentistry and Pharmaceutical Sciences, 2-5-1, Shikata-cho, Okayama 700-8558, Japan. ${ }^{2}$ Department of Molecular Hepatology, Okayama University Graduate School of Medicine, Dentistry and Pharmaceutical Sciences, Okayama, Japan. ${ }^{3}$ Health and Environment Center, Okayama University, Okayama, Japan. ${ }^{4}$ Department of Internal Medicine, Kagawa Prefectural Central Hospital, Takamatsu, Japan.

\section{Received: 19 April 2013 Accepted: 28 May 2013}

\section{Published: 1 June 2013}

\section{References}

Clark PJ, Thompson AJ, Zhu Q, Vock DM, Zhu M, Patel K, Harrison SA, Naggie S, Ge D, Tillmann HL, Urban TJ, Shianna K, Fellay J, Goodman Z, Noviello S, Pedicone LD, Afdhal N, Sulkowski M, Albrecht JK, Goldstein DB, McHutchison JG, Muir AJ (2012) The association of genetic variants with hepatic steatosis in patients with genotype 1 chronic hepatitis C infection. Dig Dis Sci 57:2213-2221

Desmet VJ, Gerber M, Hoofnagle JH, Manns M, Scheuer PJ (1994) Classification of chronic hepatitis: diagnosis, grading and staging. Hepatology 19:1513-1520

Firpi RJ, Nelson DR (2007) Current and future hepatitis C therapies. Arch Med Res 38:678-690

Ghany MG, Nelson DR, Strader DB, Thomas DL, Seeff LB, American Association for Study of Liver Diseases (2011a) An update on treatment of genotype 1 chronic hepatitis C virus infection: 2011 practice guideline by the American Association for the Study of Liver Diseases. Hepatology 54:1433-1444

Ghany MG, Nelson DR, Strader DB, Thomas DL, Seeff LB, American Association for Study of Liver D (2011b) An update on treatment of genotype 1 chronic hepatitis $C$ virus infection: 2011 practice guideline by the American Association for the Study of Liver Diseases. Hepatology 54:1433-1444

He S, McPhaul C, Li JZ, Garuti R, Kinch LN, Grishin NV et al (2009) A sequence variation (1148M) in PNPIA3 associated with nonalcoholic fatty liver disease disrupts triglyceride hydrolysis. J Biol Chem 285:6706-6715

Ikeda F, Dansako H, Nishimura G, Mori K, Kawai Y, Ariumi Y et al (2010) Amino acid substitutions of hepatitis $C$ virus core protein are not associated with intracellular antiviral response to interferon-a in vitro. Liver Int 30:1324-1331

Imai Y, Kawata S, Tamura S et al (1998) Relation of interferon therapy and hepatocellular carcinoma in patients with chronic hepatitis C. Ann Intern Med 129:94-99

Kato N (2001) Molecular virology of hepatitis C virus. Acta Med Okayama 55:133-159 Kawaguchi T, Sumida Y, Umemura A, Matsuo K, Takahashi M, Takamura T, Yasui K, Saibara T, Hashimoto E, Kawanaka M, Watanabe S, Kawata S, Imai Y, Kokubo M, Shima T, Park H, Tanaka H, Tajima K, Yamada R, Matsuda F, Okanoue T, Japan Study Group of Nonalcoholic Fatty Liver Disease (2012) Genetic polymorphisms of the human PNPLA3 gene are strongly associated with severity of non-alcoholic fatty liver disease in Japanese. PLoS One 7:e38322
Kotronen A, Johansson LE, Johansson LM, Roos C, Westerbacka J, Hamsten A et al (2009) A common variant in PNPLA3, which encodes adiponutrin, is associated with liver fat content in humans. Diabetologia 52:1056-1060

Kumada H, Toyota J, Okanoue T, Chayama K, Tsubouchi H, Hayashi N (2012) Telaprevir with peginterferon and ribavirin for treatment-naive patients chronically infected with HCV of genotype 1 in Japan. J Hepatol 56:78-84

Makuuchi M, Kokudo N, Arii S (2010) Clinical practice guidelines for hepatocellular carcinoma - the Japan society of hepatology 2009 update. Hepatol Res 40:2-144

Miyashita M, Ito T, Sakaki M, Kajiwara A, Nozawa H, Hiroishi K, Kobayashi M, Kumada H, Imawari M (2012) Genetic polymorphism in cyclooxygenase-2 promoter affects hepatic inflammation and fibrosis in patients with chronic hepatitis C. J Viral Hepat 19:608-614

Nishiguchi S, Shiomi S, Nakatani S et al (2001) Prevention of hepatocellular carcinoma in patients with chronic active hepatitis $C$ and cirrhosis. Lancet 357:196-197

Poynard T, Ratziu V, McHutchison J, Manns M, Goodman Z, Zeuzem S, Younossi Z, Albrecht J (2003) Effect of treatment with peginterferon or interferon alfa-2b and ribavirin on steatosis in patients infected with hepatitis C. Hepatology 38:75-85

Romeo S, Kozlitina J, Xing C, Pertsemlidis A, Cox D, Pennacchio LA, Boerwinkle E, Cohen JC, Hobbs HH (2008) Genetic variation in PNPLA3 confers susceptibility to nonalcoholic fatty liver disease. Nat Genet 40:1461-1465

Takeuchi Y, Ikeda F, Moritou Y, Hagihara H, Yasunaka T, Kuwaki K, Miyake Y, Ohnishi H, Nakamura S, Shiraha H, Takaki A, Iwasaki Y, Nouso K, Yamamoto K (2013) The impact of patatin-like phospholipase domain-containing protein 3 polymorphism on hepatocellular carcinoma prognosis. J Gastroenterol 48:405-412

Trépo E, Pradat P, Potthoff A, Momozawa Y, Quertinmont E, Gustot T, Lemmers A, Berthillon $P$, Amininejad L, Chevallier M, Schlué J, Kreipe $H$, Devière J, Manns M, Trépo C, Sninsky J, Wedemeyer H, Franchimont D, Moreno C (2011) Impact of patatin-like phospholipase-3 (rs738409 C > G) polymorphism on fibrosis progression and steatosis in chronic hepatitis C. Hepatology 54:60-69

Valenti L, Alisi A, Galmozzi E, Bartuli A, Del Menico B, Alterio A, Dongiovanni P, Fargion S, Nobili V (2010) I148M patatin-like phospholipase domaincontaining 3 gene variant and severity of pediatric nonalcoholic fatty liver disease. Hepatology 52:1274-1280

Valenti L, Rumi M, Galmozzi E, Aghemo A, Del Menico B, De Nicola S, Dongiovanni P, Maggioni M, Fracanzani AL, Rametta R, Colombo M, Fargion S (2011) Patatin-like phospholipase domain-containing 3 I148M polymorphism, steatosis, and liver damage in chronic hepatitis C. Hepatology 53:791-799

Valenti L, Aghemo A, Stättermayer AF, Maggioni P, De Nicola S, Motta BM, Rumi MG, Dongiovanni P, Ferenci P, Colombo M, Fargion S (2012) Implications of PNPLA3 polymorphism in chronic hepatitis $C$ patients receiving peginterferon plus ribavirin. Aliment Pharmacol Ther 35:1434-1442

Yoshida H, Shiratori Y, Moriyama M et al (1999) Interferon therapy reduces the risk for hepatocellular carcinoma: National surveillance program of cirrhotic and noncirrhotic patients with chronic hepatitis C. Ann Intern Med 131:174-181

Zeuzem S, Andreone P, Pol S, Lawitz E, Diago M, Roberts S, Focaccia R, Younossi Z, Foster GR, Horban A, Ferenci P, Nevens F, Müllhaupt B, Pockros P, Terg R, Shouval D, van Hoek B, Weiland O, Van Heeswijk R, De Meyer S, Luo D, Boogaerts G, Polo R, Picchio G, Beumont M, REALIZE Study Team (2011) Telaprevir for retreatment of HCV infection. N Engl J Med 364:2417-2428

doi:10.1186/2193-1801-2-251

Cite this article as: Moritou et al:: Predictive impact of polymorphism of PNPLA3 on HCC development after interferon therapy in Japanese patients with chronic hepatitis C. SpringerPlus 2013 2:251. 